\title{
ASSESSING THE BENEFITS OF WAVELENGTH SELECTION VS. WAVELENGTH CONVERSION IN WDM NETWORKS*
}

\author{
Nicola Andriolli, Luca Valcarenghi, and Piero Castoldi \\ Scuola Superiore Sant'Anna di Studi Universitari e di Perfezionamento, Center of Excellence \\ for Communication Networks Engineering, Pisa, Italy \\ \{nick, valcarenghi, castoldi\}@sssup.it
}

\begin{abstract}
In this paper we consider how the performance of wavelength routed networks depends on node capabilities, such as the wavelength selection and the wavelength conversion. For this purpose we propose a modified version of the shortest path algorithm for the wavelength graph (SPAWG), where "virtual links" are added for modeling the utilization and the cost of these node capabilities.

Numerical results show the performance advantages in utilizing wavelength selection instead of wavelength conversion, when only one of the two capabilities can be implemented in each network node. When wavelength conversion and wavelength selection utilization is limited to a subset of network nodes to reduce the overall network cost, results indicate that sparse wavelength selection is not as beneficial as sparse wavelength conversion.
\end{abstract}

\section{INTRODUCTION}

In the latest years, the growing demand for bandwidth in core networks has been cost effectively satisfied with the deployment of wavelength division multiplexing (WDM) systems. Currently WDM is the basis of wavelength routed optical networks (WRONs), where lightpaths (i.e. all-optical end-toend switched connections between node pairs at the wavelength granularity) are set up to generate a virtual topology, often with a higher connectivity than the physical fiber network. The lightpath establishment in WRONs involves the problem of selecting a suitable path and allocating an available wavelength

\footnotetext{
*This work has been partially supported by Italian Ministry of Education and University (MIUR) under FIRB project "Enabling platforms for high-performance computational grids oriented to scalable virtual organization (GRID.IT)" and partially by Marconi through an annual grant to Scuola Superiore Sant'Anna.
} 
for the connection: the resulting problem is referred to as the routing and wavelength assignment (RWA) problem [1]. Network performance depends on the RWA problem solution, that in turn is affected by the network node capabilities.

In this paper two node capabilities have been investigated: (i) the possibility for a connection request of choosing any admission wavelength in source and destination nodes (wavelength selection, whose benefits have not been extensively studied in literature [2,3]); (ii) the possibility of converting the wavelength of the lightpath at any intermediate node (wavelength conversion, which has been thoroughly investigated [4-8]). To take into account these additional node capabilities the Shortest Path Algorithm for the Wavelength Graph (SPAWG for short $[9,10]$ ) has been extended: "virtual links" are added for modeling the utilization and the cost of any processing incurred in lightpath routing besides the hop cost. Therefore lightpaths are routed along the least cost path, that jointly minimizes the utilization of node capabilities and link capacity.

Numerical results show that, if implemented at all network nodes (fullmode), wavelength selection is more beneficial than wavelength conversion. On the other hand, if selection capabilities or conversion capabilities are deployed just in a limited subset of nodes (sparse-mode), sparse wavelength selection is not as beneficial as sparse wavelength conversion.

\section{NODE ARCHITECTURE AND RWA ALGORITHM}

Node architectures incorporating various node capabilities have been investigated. As shown in Fig. 1, wavelength selection, which allows an outgoing (incoming) request to choose the wavelength on the first (last) hop of the lightpath, is implemented connecting with a space matrix (called wavelength selection switching fabric) the tributaries to the bank of fixed-wavelength transponders [11]. This space matrix switches the traffic from the tributaries (at the wavelength $\lambda_{t}$, usually at $1300 \mathrm{~nm}$ ) to the transponder at the desired wavelength, e.g., $\lambda_{1}$ (in the $1550 \mathrm{~nm}$ range, according to ITU-T grids). On the contrary, if wavelength selection is not present, each tributary is statically linked to a fixed-wavelength transponder and it must use only a specific wavelength.

Wavelength conversion permits to change the wavelength on which a lightpath ${ }^{1}$ is carried (relaxing the wavelength continuity constraint): in Fig. 1 this capability is hidden in the optical cross-connect. To preserve signal transparency and contain complexity and power consumption [5], an all-optical wavelength conversion scheme has been adopted, assuming that only half of

\footnotetext{
${ }^{1}$ We assume that wavelength conversion in a node cannot be performed for lightpaths added or dropped in the same node because this function is provided through wavelength selection.
} 
the wavelengths nearest to the working one can be reached, due to technological constraints (previous studies have shown that this limitation only slightly affects network performance [6]).

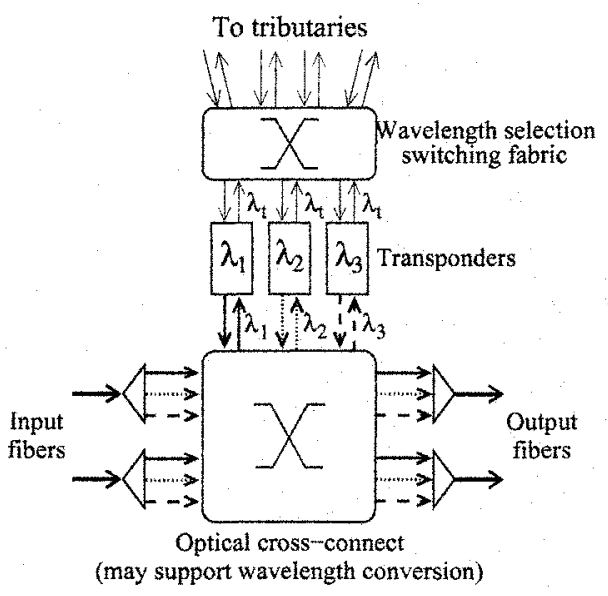

Figure 1. Architecture of an optical node with $W=$ 3 wavelengths per link and nodal degree $D=2$.

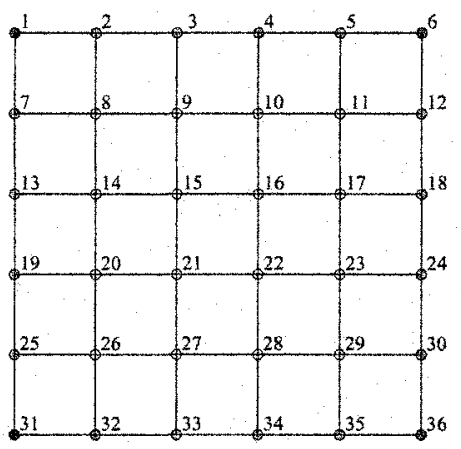

Figure 2. $6 \times 6$ grid topology.

To consider both wavelength selection and wavelength conversion costs, an auxiliary graph, called wavelength graph (WG) [9], is utilized by the SPAWG routing and wavelength assignment algorithm to find a path for the incoming connection requests. The WG is built repeating the physical topology of the network on a number of superimposed planes equal to the number of available wavelengths. On each layer the links connecting the nodes are assigned a weight that is a measure of the hop cost.

To consider the wavelength conversion, "virtual links" are added among copies of the same node on different layers. Due to the high cost and the physical constraints of the wavelength converters, the weight of these links is an order of magnitude higher than the hop cost, and proportional to the distance between wavelengths. To take into account the wavelength selection, a modified version of the WG is adopted, with an additional layer $\lambda_{0}$ : this is a logical level that contains only the network nodes without links among them. Instead, there are "virtual links" that connect source and destination nodes of a connection request with their respective copies on one or more layers, accordingly to the absence or presence of wavelength selection. Since wavelength selection is less complex and expensive than wavelength conversion, the weight of these links has the same order of magnitude of the hop cost. 


\section{NETWORK AND TRAFFIC MODEL}

A $6 \times 6$ grid topology with bidirectional links and $W$ wavelengths per link, depicted in Fig. 2, is utilized as test network. The grid topology is deployed in grid computing scenarios, and it has been chosen because its simplicity and regularity allow to clearly identify the individual effects of various parameters on network performance [12] (results on other topologies, not shown here for brevity, confirmed the conclusions drawn on the grid network).

Traffic between node pairs is generated with uniform probability: $T$ represents the average number of connections requested by every node. An incremental traffic model has been adopted: lightpath requests arrive sequentially and, if accepted, remain in the network indefinitely. This approach resembles the present connection deployment procedures, where the lightpaths are established upon a customer request and remain active for long periods of time (months to years).

If wavelength selection is not present, for each connection request a pair of transponders is also randomly chosen in source and in destination nodes. Only these transponders can be exploited by the tributaries. In case of absence of both wavelength selection and wavelength conversion two transponders with the same wavelength are chosen ${ }^{2}$.

The lightpath requests are processed upon arrival by the SPAWG algorithm. If a minimum cost route that connects source and destination nodes is found a bidirectional lightpath is established, and the network state is updated; otherwise the connection is blocked and rejected.

\section{NUMERICAL RESULTS}

The metric considered to evaluate network performance is the blocking probability, computed as the ratio between the number of blocked lightpath requests and the number of generated requests.

In the first part of the section we compare the four architectures obtained combining the absence or the presence of both wavelength selection (WS) and wavelength conversion (WC) in each network node: NOWS-NOWC, NOWSWC, WS-NOWC, and WS-WC.

In the second part of the section sparse wavelength selection and conversion are considered: in this case, the placement of these node capabilities must be optimized. The optimal placement problem has been proven to be NPcomplete [7]. For this reason the following heuristic [8] has been utilized:

\footnotetext{
${ }^{2}$ Differently from [2], where networks with fixed transmitters and receivers and absence of wavelength conversion are not studied because full connectivity is not guaranteed, we consider this case (adding the condition that the two transponders work on the same wavelength), because this scenario is typical of many present optical networks.
} 


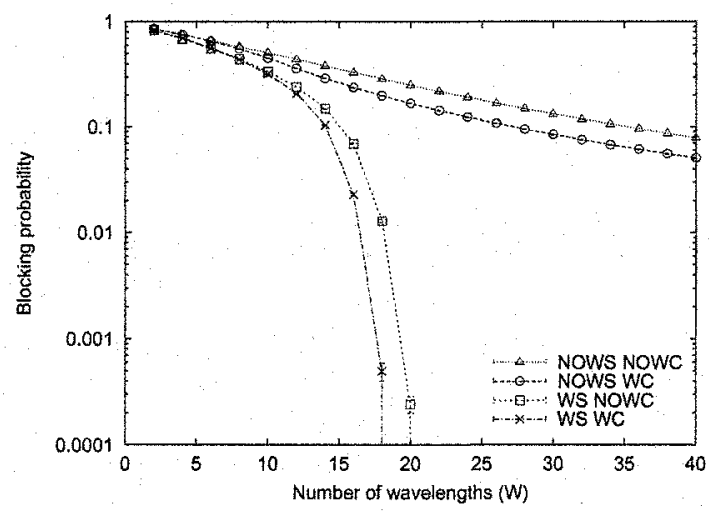

Figure 3. Blocking probability versus wavelengths per link, with $T=10$ calls per node.

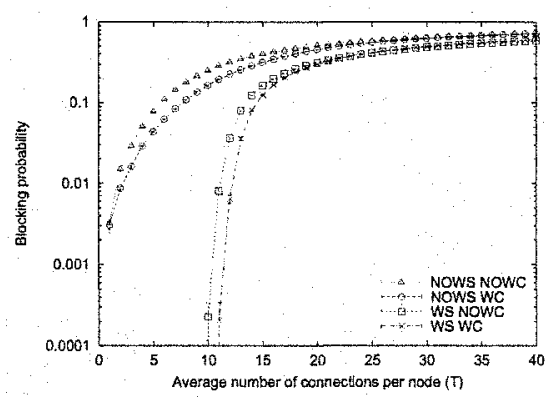

(a) $W=20$

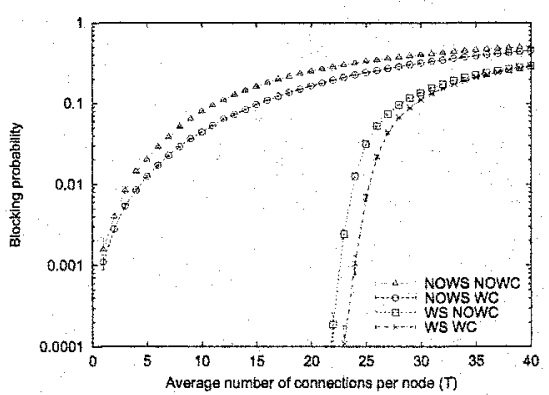

(b) $W=40$

Figure 4. Blocking probability versus offered traffic.

we start from a scenario where the capability is present in all the nodes and we rank them according to its utilization; then simulations are run placing the capability only where it is significantly used.

In all the simulations at least 5000 repetitions have been run for each point; the results are plotted with the confidence interval at $95 \%$ confidence level.

\subsection{Presence vs. absence of wavelength selection and conversion}

Fig. 3 compares the performance of the four basic node architectures, NOWSNOWC, NOWS-WC, WS-NOWC, and WS-WC, in terms of blocking probability as a function of the number of wavelengths $W$; the average number of connections $T$ requested by every node is set to 10 . This plot shows the fundamental role of wavelength selection to improve network performance, while the introduction of wavelength conversion offers only a marginal blocking de- 
crease. Indeed wavelength selection greatly simplifies the search for a suitable lightpath by allowing a flexible admission in the network. On the contrary in absence of selection lightpath requests cannot easily enter the network because, in the first and last hop, only one wavelength can be used. Architectures without wavelength selection show poor performance also in terms of scalability: increasing the number of channels per link the reduction of the blocking probability is very limited.

Fig. 4-(a) and 4-(b) show the blocking probability of the four node architectures as a function of the offered traffic for $W=20$ and $W=40$ respectively. Again the WS-WC architecture obtains the lowest blocking probability while the NOWS-NOWC architecture shows the highest blocking probability. Moreover the implementation of just wavelength conversion capabilities in the network nodes does not noticeably improve the blocking probability with respect to the NOWS-NOWC architecture. Instead wavelength selection determines a much larger improvement than the wavelength conversion, which becomes more remarkable when $W$ is increased from 20 to 40 , because the advantages of selection are augmented by the possibility of choosing a wavelength in a larger set of wavelengths.

\subsection{Sparse wavelength selection and conversion}

Fig. 5 shows the number of selections and conversions in each node of the $6 \times 6$ grid topology with $W=40$ and $T=40$. Wavelength selection is more exploited at the network edges, while wavelength conversion is mainly used in the core. Both effects are due to the fact that the central region of the network is more loaded than the edges. Wavelength selection cannot be fully exploited in the inner nodes because link congestion causes a higher blocking of connections leaving or entering those nodes. However this capability is widely used in the entire network: more than 16 selections take place in every node. On the other hand wavelength conversion helps to find a route for requests that would otherwise be blocked because of the wavelength continuity constraint: hence this capability is much more exploited in the central region of the network where the majority of the traffic is routed (more than 10 conversions occur), while it is only marginally used at the edges (from 3 to 5 ).

In Fig. 6 the blocking probability versus offered traffic is shown with sparse wavelength selection and conversion. We assign WS and WC capabilities only to the first $q_{W S}$ and $q_{W C}$ fraction of the total number of nodes, in the ranking of WS and WC utilization. In particular two wavelength conversion scenarios $\left(q_{W C} \in\{0.25,1\}\right)$ are combined with three wavelength selection scenarios $\left(q_{W S} \in\{0,0.5,1\}\right.$ ). From the figure we can notice that sparse wavelength selection $\left(q_{W S}=0.5\right.$ ) only slightly reduces the blocking probability with respect to the case without wavelength selection $\left(q_{W S}=0\right)$, but significantly increases 


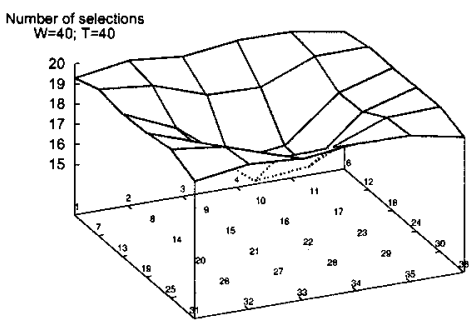

(a) Wavelength selection

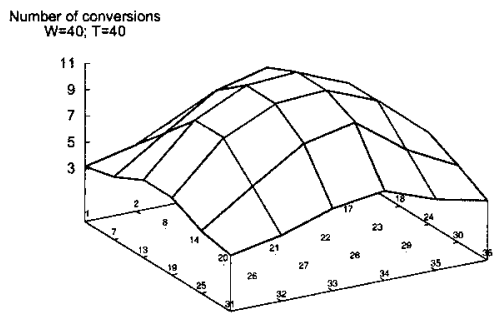

(b) Wavelength conversion

Figure 5. Number of selections and conversion in each node of the $6 \times 6$ grid topology.

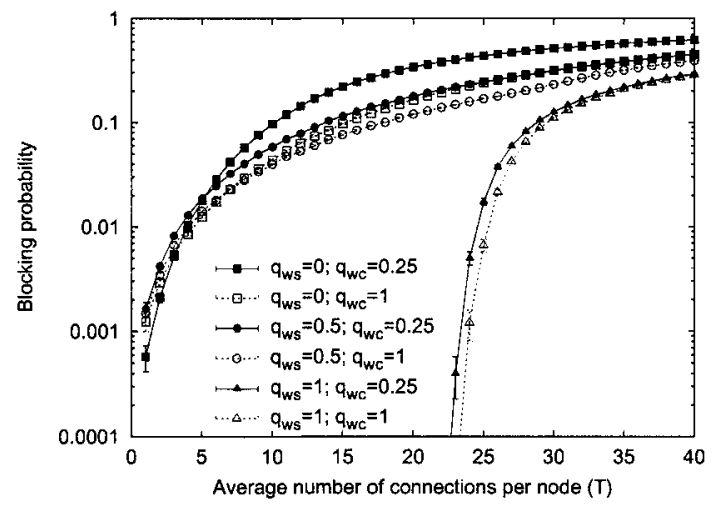

Figure 6. Blocking probability versus offered traffic for sparse WS and WC $(W=40)$.

it with respect to the case in which wavelength selection is available in all network nodes $\left(q_{W S}=1\right)$. Indeed wavelength selection simplifies very much the search of an available lightpath, but it is a property of each specific optical node, which can be exploited only by the tributaries connected to that node. Thus it does not guarantee a fair improvement of the network performance.

On the contrary, the assignment of wavelength conversion capability to just one-fourth of the nodes ( $q_{W C}=0.25$ ) closely approximates the blocking probability obtained when wavelength conversion capability is present in all network nodes $\left(q_{W C}=1\right)$. This happens because even sparse wavelength conversion can be effectively utilized by all the connections traversing a node equipped with this capability, increasing in a fair manner the network performance. Thus, unlike wavelength selection, wavelength conversion provides a reduction in blocking probability approximately proportional to its exploitation. 


\section{CONCLUSION}

In this paper we evaluated the benefits of enhanced node capabilities in wavelength routed networks. Numerical results have shown the superiority and criticality of wavelength selection. Very low blocking probability can be obtained only if wavelength selection is present in all network nodes. On the contrary, full and sparse wavelength conversion give a similar and moderate performance improvement.

In fact, only the connections admitted in nodes with wavelength selection can exploit this capability: then the network can leverage wavelength selection only if present in all the nodes. On the contrary, wavelength conversion provided in a node can be exploited by all the connections traversing that node. In this sense wavelength conversion, though placed in nodes, is a shared facility while wavelength selection is a node-peculiar facility.

\section{REFERENCES}

[1] H. Zang, J. P. Jue, and B. Mukherjee, "A review of routing and wavelength assignment approaches for wavelength-routed optical WDM networks," Optical Networks Magazine, vol. 1, pp. 47-60, Jan. 2000.

[2] J. Yates, J. Lacey, M. Rumsewicz, and M. Summerfield, "Performance of networks using wavelength conversion based on four-wave mixing in semiconductor optical amplifiers," J. Lightwave Technol., vol. 17, pp. 782-791, May 1999.

[3] I. Widjaja and A. I. Elwalid, "Study of GMPLS lightpath setup over lambda-router networks," in Proc. IEEE ICC'02, vol. 5, pp. 2707-2711, 2002.

[4] J. Yates, J. Lacey, and M. Rumsewicz, "Wavelength converters in dynamically reconfigurable WDM networks," IEEE Commun. Surveys, vol. 2, $2^{\text {nd }}$ quarter 1999. Available at http://www.comsoc.org/pubs/surveys/.

[5] B. Ramamurthy and B. Mukherjee, "Wavelength conversion in WDM networking," IEEE J. Select. Areas Commun., vol. 16, pp. 1061-1073, Sep. 1998.

[6] J. Yates, J. Lacey, D. Everitt, and M. Summerfield, "Limited-range wavelength translation in all-optical networks," in Proc. IEEE INFOCOM'96, vol. 3, pp. 954-961, 1996.

[7] G. Wilfong and P. Winkler, "Ring routing and wavelength translation," in Proc. ACMSIAM Symposium on Discrete Algorithms (SODA), 1998.

[8] K. Venugopal, M. Shivakumar, and P. Kumar, "A heuristic for placement of limited range wavelength converters in all-optical networks," in Proc. IEEE INFOCOM'99, vol. 2, pp. 908-915, 1999.

[9] I. Chlamtac, A. Farago, and T. Zhang, "Lightpath (wavelength) routing in large WDM networks," IEEE J. Select. Areas Commun., vol. 14, pp. 909-913, Jun. 1996.

[10] D. Saha, "Lightpath versus semi-lightpath: some studies on optimal routing in WDM optical networks," Photonic Network Commun., Kluwer, vol. 2, pp. 155-161, May 2000.

[11] R. Ramaswami and K. N. Sivarajan, Optical Networks: a practical perspective. Morgan Kaufmann, second ed., 2002.

[12] D. K. Hunter, E. D. Lowe, and I. Andonovic, "Modelling of routing in multi-fibre WDM grid networks," in Proc. IEE Colloquium on WDM Technol. and Applic., 1997. 